\title{
OPTIMIZED MODEL-FUNCTIONS FOR CCD-CAMERA BASED ASTROMETRIC PLATE MEASUREMENTS
}

\author{
L. WINTER \\ Hamburger Sternwarte \\ Gojenbergsweg 112 \\ D-21029 Hamburg \\ Germany
}

\begin{abstract}
While digitizing the first 1500 photographic plates (astrograph plates and prime focus plates of various telescopes) using our CCD-based measuring machine, we gained a detailed understanding of the properties of the digitized stellar images. These images are different from those obtained by digitizing photographic plates with microdensitometers and similar scanners. Therefore it was necessary to reject the modelling methods given in the literature and compose new models that describe the stellar images more accurately.

First results of simulating the whole imaging process, starting with the atmosphere, aberrations of the telescope, etc., and ending by including the imaging-properties of our measuring machine, show good agreement with the measured images as well as the modelling functions.
\end{abstract}

\section{Introduction}

This paper presents some results of a detailed investigation concerning centring methods as applied in astrometry. Our main goal was a high accuracy of the image centre, whereas other properties (i.e. photometry) could be neglected.

Assuming circular symmetry for all stellar images, one can think of the image-function $I(x, y)$ that is the image as seen by the CCD-camera, having a centre at $\left(\mathrm{x}_{0}, \mathrm{y}_{0}\right)$, to be separable into a geometric part $r=\left(\left(x-x_{0}\right)^{2}+\left(y-y_{0}\right)^{2}\right)^{1 / 2}$ and an intensity part $\mathrm{I}(\mathrm{r})$. Thus $\mathrm{I}(\mathrm{x}, \mathrm{y}) \rightarrow \mathrm{I}(\mathrm{r})$ and $r=r(x, y)$.

Our measuring engine measures the transmittance of a photographic plate. Therefore we chose $P(r)=1-\left(I(r) / I_{0}\right)$ as the normalized profile-function, $I_{0}$ being the intensity of the illumination. Thus the peak of profile $P(r)=1$ represents the maximum intensity of the stellar image as seen on the sky.

\section{Empirical Investigation}

Figure 1 shows measured stellar profiles of a magnitude sequence in NGC 6791 using our measuring engine HAM 1. It is obvious that for all but the faintest stars (i.e. V $>14.0 \mathrm{mag}$ ) the image-profiles look similar and can be modelled by the same profile-function $P(r)$. 


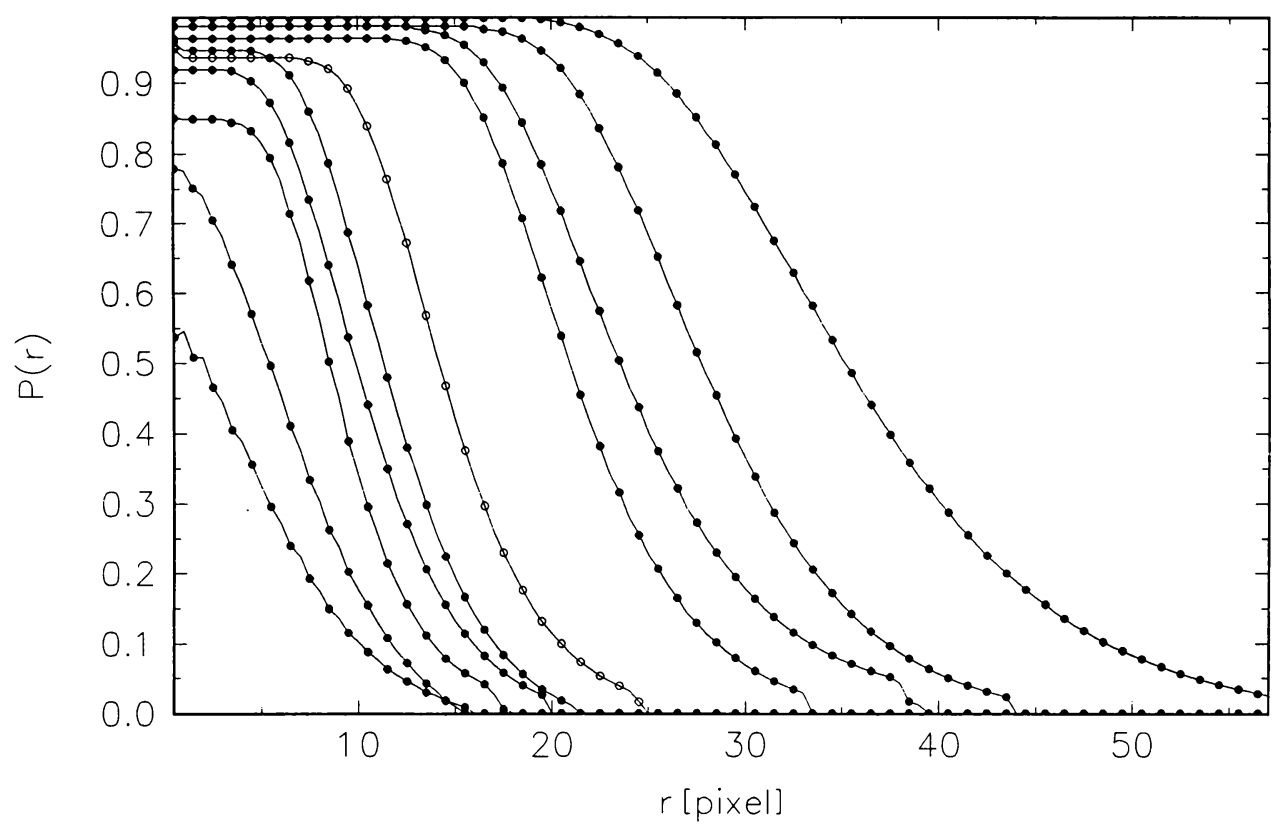

Figure 1. Magnitude sequence. The dots are measured data, and the lines are modelled data.

\section{Approximation of Empirical Profiles}

Our first idea was to find an analytical function $f(x)$ having similar properties as $P(r)$. These properties are:

1. amplitude of $P(r)$ nearly independent of magnitude

2. background noise similar for all pictures

3. for $\mathrm{r} \rightarrow 0 \mathrm{P}(\mathrm{r}) \rightarrow 1$

4. for $r \rightarrow \infty P(r) \rightarrow 0$

5. gradient in $r_{1 / 2}$ varies only slightly with $V$, where $P\left(r_{1 / 2}\right)=0.5$.

Some usable functions are e.g. ARCTAN or TANH. If a simple transformation is used:

$$
M(r)=a \cdot f\left(r-r_{y_{2}}\right)+b \text {, }
$$

$M(r)$ being the modelling function. Taking into account the variation in the gradient as well, we get:

$$
M(r)=a \cdot f\left(s\left(r-r_{y s}\right)\right)+b,
$$

where $f$ is one of the functions mentioned above, and $a, b, s$ are parameters of the model $M(r)$.

A further improvement can be achieved by modelling the upper and lower part of the profile separately, by constructing a function $f(x)$ out of two separate functions $f_{1}(x)$ and $f_{2}(x)$ having a different asymptotical behaviour. The Fermi-Dirac-distribution function is an example, but is similar to TANH. (For a comparison with the stellar profile-functions, see Winter et al. 1992).

The lines in Fig. 1 show an example of a new model constructed in this way (using the computed parameters for the magnitude sequence). 


\section{Simulation}

Our latest investigation is a simulation of the imaging process somewhat similar to Moffat's approach (Moffat 1969) but takes into account the properties of our astrograph and measuring engine. The resulting profile-functions are in good agreement with our measurements, and they look almost identical to the model given in Fig.1.

The only property of the imaging system not yet modelled is the behaviour of the camera electronics. An investigation showed an asymmetry in the $\mathrm{X}$ and $\mathrm{Y}$ axis, as well as a lowpass filter characteristic in the $\mathrm{X}$ direction.

\section{References}

Moffat, A.F.J., 1969. 'Theoretical investigation of focal stellar images in the photographic emulsion and application to photographic photometry', Astron. \& Astrophys., 3, 455.

Winter, L., de Vegt, Chr., Steinbach, M. and Zacharias, N., 1992. 'Hardware and software aspects of CCD camera-based astrometric plate measurements', in 'Digitised Optical Sky Surveys', eds. H.T. MacGillivray and E.B. Thomson, Kluwer, Dordrecht, p. 123. 